\title{
Minimal residual disease after radical surgery in EGFR-mutant non-small cell lung cancer
}

\author{
Katsuhiro Masago ${ }^{1}$, Yoshitsugu Horio ${ }^{2}$, Shiro Fujita ${ }^{1}$, Yasushi Yatabe ${ }^{1,3}$ \\ ${ }^{1}$ Department of Pathology and Molecular Diagnostics, ${ }^{2}$ Department of Thoracic Oncology, Aichi Cancer Center Hospital, Aichi, Japan; ${ }^{3}$ Department \\ of Diagnostic Pathology, National Cancer Center Hospital, Tokyo, Japan \\ Correspondence to: Katsuhiro Masago, MD, PhD. Department of Pathology and Molecular Diagnostics, Aichi Cancer Center Hospital, 1-1 \\ Kanokoden, Chigusa-ku, Nagoya City, Aichi 4648681, Japan. Email: masago@aichi-cc.jp. \\ Provenance: This is an invited article commissioned by the Academic Editor Dr. Xianglin Hu (Department of Pulmonary Medicine, Zhongshan \\ Hospital, Fudan University, Shanghai, China). \\ Comment on: Xu ST, Xi JJ, Zhong WZ, et al. The Unique Spatial-Temporal Treatment Failure Patterns of Adjuvant Gefitinib Therapy: A Post Hoc \\ Analysis of the ADJUVANT Trial (CTONG 1104). J Thorac Oncol 2019;14:503-12.
}

Submitted Aug 19, 2019. Accepted for publication Sep 05, 2019.

doi: $10.21037 /$ tlcr.2019.09.09

View this article at: http://dx.doi.org/10.21037/tlcr.2019.09.09

$\mathrm{Xu}$ et al. performed a post-hoc analysis of a randomized phase III trial (ADJUVANT/CTONG1104) of adjuvant gefitinib therapy in the treatment of Chinese patients who had undergone complete resection for EGFR-mutant stage II-IIIA non-small cell lung cancer (NSCLC) to evaluate patterns of spatial-temporal treatment-failure $(1,2)$. Two hundred twenty-two patients were randomized 1:1 to receive gefitinib or vinorelbine plus cisplatin (VP). Among them, 106 patients received gefitinib treatment, and 87 patients who received VP completed 4 cycles of chemotherapy. One hundred twenty-four patients experienced disease progression during a median follow-up period of 36.5 months.

A spatial distribution analysis revealed that the most frequent site of metastasis was the central nervous system (CNS) in the gefitinib group $(27.4 \%)$ and extracranial sites in the VP group (36.8\%). A temporal distribution analysis revealed that the rate of recurrence was lower in the gefitinib group in the early period after surgery. The first peak of extracranial metastasis in the VP and gefitinib groups appeared at 9-15 and 24-30 months, respectively. After surgery, the highest peak for CNS metastasis in the VP and gefitinib groups occurred at $12-18$ and 24-36 months, respectively.

In this post-hoc analysis report, the temporal recurrence patterns were analyzed based on hazard ratios, an analytical method that may be applicable to the post-hoc analysis of data from other clinical trials. However, there are several points that need to be noted when interpreting the results.

The median disease-free survival (DFS) of patients with extracranial metastasis was significantly better in the gefitinib group. There was no significant between-arm difference in the DFS of patients with CNS metastasis, although metastasis was first seen in the CNS more frequently in the gefitinib group. This may be cerebrospinal fluid concentrations of gefitinib remain low due to poor penetration (3), although the same is also true for VP $(4,5)$. Considering that osimertinib, a $3^{\text {rd }}$ generation EGFR-TKI, showed better efficacy in the first-line treatment of EGFRmutant advanced NSCLC in the FLAURA trial (6), it is also likely that later generation EGFR-TKIs, including osimertinib, are in the same situation (7), as the authors also mentioned in this report.

The temporal hazard ratio shows the temporal features of the recurrence patterns in each arm. The chemotherapy group showed a rapid increase in the rate of recurrence at $9-15$ months after surgery. Adjuvant VP is completed from 12 weeks after surgery, and the rate of recurrence at approximately 1 year after surgery can thereafter be judged to be slightly better in comparison to advanced NSCLC patients who receive platinum-doublet therapy (8), considering that the residual disease is originally minimal and that it takes a relatively long time to detect recurrent lesions in imaging studies. In addition, the first peak of 
Table 1 List of clinical trials with EGFR-TKI for postoperative adjuvant therapy

\begin{tabular}{|c|c|c|c|c|}
\hline Authors & $\begin{array}{l}\text { Median follow-up } \\
\text { time (months) }\end{array}$ & $\begin{array}{c}\text { Median DFS } \\
\text { time (total) (months) }\end{array}$ & $\begin{array}{l}\text { Median DFS time } \\
\text { (central nervous system) (months) }\end{array}$ & $\begin{array}{c}\text { Median DFS time } \\
\text { (extra-cranial) (months) }\end{array}$ \\
\hline Xu et al. (2) & 36.5 & 28.7 (Gefitinib) & 40.8 (Gefitinib) & 45.2 (Gefitinib) \\
\hline Kelly et al. (12) & 47 & 50.5 (Erlotinib) & NA & NA \\
\hline Pennel et al. (13) & & 28.5 (Placebo) & NA & NA \\
\hline Pigmon et al. (11) & 62.4 & $\begin{array}{c}{ }^{*} 5.3 \% \text { improvement of DFS at } \\
5 \text { years (platinum-doublet) }\end{array}$ & NA & NA \\
\hline
\end{tabular}

*, data of the median DFS not available. DFS, disease-free survival; NA, not applicable; TKI, tyrosine kinase inhibitor; VP, cisplatin and vinorelbine.

extracranial metastasis at $9-15$ months in patients treated with VP indicates the presence of subclones that cannot be suppressed by VP therapy, and additional subclones may be present in EGFR mutant cells. The analysis of the clinical background and precise patterns of recurrence in patients with extracranial metastasis who relapse at 9-15 months may provide some suggestive insights. Furthermore, these differences in peaks may be partially due to the duration of treatment. In the gefitinib group, patients began to relapse after 12 months, which is reasonable, considering that the median progression-free survival (PFS) of gefitinibtreated advanced EFGR-mutant lung cancer patients is approximately 10 months $(9,10)$. In addition, as mentioned above, the period of 12 months is considered reasonable as the target lesions are minimal.

In the Kaplan-Meier curves for DFS, not-very-sharp changes can be seen in the time course. In the protocol, chest CT and chest radiography were performed in turn every three months, while brain MRI and a bone scan by emission CT were performed every 6 and 12 months, respectively. In many cases, recurrence is detected in imaging studies; thus, it is typical for these Kaplan-Meier curves to show a stair-like pattern. The true tendency of recurrence may be masked by these step-like shapes. Thus, the dynamics of the hazard rates for recurrence can help to clarify the temporal tendency for recurrence, and such analyses should be applied to the evaluation of the temporal recurrence patterns of other clinical trials, including posthoc analyses.

The present study was associated with several limitations. First, the observation period was relatively short. In addition, the 3-year DFS rates of the gefitinib and VP groups were $34 \%$ and $27 \%$, respectively, which are relatively poor in comparison to studies performed in similar settings (11). This may indicate some contradictions of the inclusion criteria (Table 1). The usefulness of postoperative gefitinib therapy in the elimination of minimal residual disease (MRD) may therefore be clarified by excluding patients with N2 disease from the cohort. The imaging modalities that were applied for the staging of patients in the gefitinib arm were CT (59\%), PET-CT (24\%), and MRI or another imaging modality (16\%). Those that were applied for the staging of the patients in the VP group were CT (58\%), PET-CT (22\%), and MRI or another type of modality (21\%).

In terms of patient selection, the prescribed treatment was completed by 106 patients in the gefitinib group and 87 patients in the VP group. At this point, the analysis of the differences in the events of each of the in which recurrence was observed (gefitinib, n=58; VP, n=56) has already resulted in a significantly biased cohort.

In this ADJUVANT trial, the EGFR T790M status of patients in the gefitinib group who relapsed after surgery remains unknown. Thus, it may be necessary to compare gefitinib adjuvant therapy in patients who receive platinumbased adjuvant therapy and in whom EGFR-TKI therapy is administered at the time of relapse to ascertain the influence of T790M on the treatment strategy of the ADJUVANT trial. In addition, T790M has also been reported to be associated with a more indolent phenotype (14), which may have led to the relatively constant increase in the HR of the gefitinib group.

Since MRD with no clinical signs of recurrences after 
surgery is associated with tumor recurrence, future studies should focus on how to capture postoperative MRD. Since it is difficult to capture MRD at the point when it appears in follow-up imaging studies, as mentioned in this text, it is recommended that circulating tumor DNA (ctDNA) be used to detect tumor-derived miRNA or driver genes by high-sensitivity PCR (e.g., digital-PCR). There are two major problems. The first problem is that it is not clear which commercial kit is appropriate for collecting ctDNA. These kits include the widely used the cobas ${ }^{\circledR}$ EGFR Mutation Test v2 (cobas plasma) assay (Roche Molecular Systems, Inc., Pleasanton, CA, USA), which was adopted in FLAURA study (15), the QIAamp ${ }^{\circledR}$ circulating nucleic acid kit (Qiagen, Hilden, Germany) and Maxwell RSC ${ }^{\circledR}$ ccfDNA plasma kit (Promega, Madison, WI, USA). In contrast, there is no consensus regarding the superiority of plasma exosomal DNA over ctDNA (16). The second problem is that the procedures that are most appropriate for detecting MRD remain unclear. This is an emerging clinical question that is currently under study (17). Furthermore, performing therapeutic intervention based solely on the results of highsensitivity tests in situations where false positives are an issue remains difficult, and this can also lead to difficulties in treating MRD.

The strongest evidence that the eradication of MRD can prevent relapse-as a measurable surrogate for a curecomes from experience in the administration of adjuvant therapy to patients with completely resected NSCLC, which is fundamentally intended to eradicate MRD that escapes surgical resection (18). However, whether or not MRD below the limit of detection indicates a cure remains unclear. In addition, since neither chemotherapy nor molecular-targeted therapy can be said to achieve total cell elimination in patients with advanced malignant epithelial tumors, it remains unclear whether EGFR-TKIs should be administered in an adjuvant setting or at the time of relapse in patients with stage II-IIIA NSCLC. These issues may suggest that the purpose of adjuvant therapy is to keep the tumor small and the tumor cells dormant while continuing to perform immune surveillance.

In terms of medical economics, the use of a sensitive method that is relatively inexpensive and easy to implement is more desirable than the conventional follow-up strategy of repeated monitoring by expensive imaging studies. Although it is important to detect MRD as early as possible after surgery, it is also important to capture postoperative MRD quantitatively. Thus, postoperative adjuvant chemotherapy for the purpose of total cell killing to prevent
MRD after surgery may become unnecessary.

Ultimately, two important clinical questions need to be clarified when defining MRD. The first question is: to what degree can MRD be completely cured by drug therapy alone? Based on the clinical observations made thus far, when the lesion is of a size that can be detected on radiographic imaging, it is widely taken as a fact that drug therapy alone will be unable to cure the disease. Thus, the level at which medical treatment can cure residual disease is defined as a certain value of some high-sensitivity index that shows the presence of lesions such as ctDNA when imaging studies show no viable lesions. The second question is: in what situation would local radical treatment lead to a complete cure in cases of postoperative recurrence? The answer to this question will be based on the size and number of lesions and the organ in which the lesion exists, a condition in which a high-sensitivity index can indicate the presence of the lesion does not exceed a certain numerical value. These clinical questions are the motivation for the setting the definition of MRD, which will be formed via worldwide consensus over time.

In the present study, if the imaging evaluation period had been set to once every 2 months in this protocol, for the sole purpose of more precisely analyzing the temporal HR, the shape of the HR curve might have been different. In other words, it is important to for the timing of imaging evaluations to be set appropriately and to ensure that there is no difference in the timing of evaluation between groups. It is therefore necessary to verify whether the increase in the hazard ratio for recurrence in the gefitinib group that was observed at 21 and 30 months after surgery reflects any biological factors, or whether it is simply a numerical value that changes significantly with changes in the timing of followup imaging evaluations. In this study, although the method for generating the temporal hazard plot was remarkable, the evaluation had to be carefully performed, as these results can change depending on the imaging timing of evaluation.

The results of the analysis will reflect features more accurately when complete data have been gathered after a sufficient follow-up period.

\section{Acknowledgments}

None.

\section{Footnote}

Conflicts of Interest: The authors have no conflicts of interest 
to declare.

Ethical Statement: The authors are accountable for all aspects of the work in ensuring that questions related to the accuracy or integrity of any part of the work are appropriately investigated and resolved.

\section{References}

1. Zhong WZ, Wang Q, Mao WM, et al. Gefitinib versus vinorelbine plus cisplatin as adjuvant treatment for stage II-IIIA (N1-N2) EGFR-mutant NSCLC (ADJUVANT/ CTONG1104): a randomised, open-label, phase 3 study. Lancet Oncol 2018;19:139-48.

2. Xu ST, Xi JJ, Zhong WZ, et al. The Unique SpatialTemporal Treatment Failure Patterns of Adjuvant Gefitinib Therapy: A Post Hoc Analysis of the ADJUVANT Trial (CTONG 1104). J Thorac Oncol 2019;14:503-12.

3. Togashi Y, Masago K, Masuda S, et al. Cerebrospinal fluid concentration of gefitinib and erlotinib in patients with non-small cell lung cancer. Cancer Chemother Pharmacol 2012;70:399-405.

4. Mouchard-Delmas C, Gourdier B, Vistelle R, et al. Modification of the blood-brain barrier permeability by vinorelbine: effect of intracarotid infusion compared with intravenous infusion. Anticancer Drugs 1996;7:213-9.

5. Laurell G, Andersson A, Engstrom B, et al. Distribution of cisplatin in perilymph and cerebrospinal fluid after intravenous administration in the guinea pig. Cancer Chemother Pharmacol 1995;36:83-6.

6. Soria JC, Ohe Y, Vansteenkiste J, et al. Osimertinib in Untreated EGFR-Mutated Advanced Non-Small-Cell Lung Cancer. N Engl J Med 2018;378:113-25.

7. Tazza M, Metro G. Adjuvant treatment of non-small cell lung cancer: focus on targeted therapy. J Thorac Dis 2017;9:4064-9.

8. Smit EF, van Meerbeeck JP, Lianes P, et al. Three-arm randomized study of two cisplatin-based regimens and paclitaxel plus gemcitabine in advanced non-small-cell lung cancer: a phase III trial of the European Organization for Research and Treatment of Cancer Lung Cancer Group--EORTC 08975. J Clin Oncol 2003;21:3909-17.

9. Sequist LV, Martins RG, Spigel D, et al. First-line gefitinib in patients with advanced non-small-cell lung cancer harboring somatic EGFR mutations. J Clin Oncol 2008;26:2442-9.

10. Mok TS, Wu YL, Thongprasert S, et al. Gefitinib or carboplatin-paclitaxel in pulmonary adenocarcinoma. $\mathrm{N}$ Engl J Med 2009;361:947-57.

11. Pignon JP, Tribodet H, Scagliotti GV, et al. Lung adjuvant cisplatin evaluation: a pooled analysis by the LACE Collaborative Group. J Clin Oncol 2008;26:3552-9.

12. Kelly K, Altorki NK, Eberhardt WE, et al. Adjuvant Erlotinib Versus Placebo in Patients With Stage IBIIIA Non-Small-Cell Lung Cancer (RADIANT): A Randomized, Double-Blind, Phase III Trial. J Clin Oncol 2015;33:4007-14.

13. Pennell NA, Neal JW, Chaft JE, et al. SELECT: A Phase II Trial of Adjuvant Erlotinib in Patients With Resected Epidermal Growth Factor Receptor-Mutant Non-SmallCell Lung Cancer. J Clin Oncol 2019;37:97-104.

14. Oxnard GR, Arcila ME, Chmielecki J, et al. New strategies in overcoming acquired resistance to epidermal growth factor receptor tyrosine kinase inhibitors in lung cancer. Clin Cancer Res 2011;17:5530-7.

15. Gray JE, Okamoto I, Sriuranpong V, et al. Tissue and Plasma EGFR Mutation Analysis in the FLAURA Trial: Osimertinib vs Comparator EGFR-TKI as First-Line Treatment in Patients with EGFR Mutated Advanced NSCLC. Clin Cancer Res 2019.

16. Möhrmann L, Huang HJ, Hong DS, et al. Liquid Biopsies Using Plasma Exosomal Nucleic Acids and Plasma CellFree DNA Compared with Clinical Outcomes of Patients with Advanced Cancers. Clin Cancer Res 2018;24:181-8.

17. Chae YK, Oh MS. Detection of Minimal Residual Disease Using ctDNA in Lung Cancer: Current Evidence and Future Directions. J Thorac Oncol 2019;14:16-24.

18. Winton T, Livingston R, Johnson D, et al. Vinorelbine plus cisplatin vs. observation in resected non-small-cell lung cancer. N Engl J Med 2005;352:2589-97.

Cite this article as: Masago K, Horio Y, Fujita S, Yatabe Y. Minimal residual disease after radical surgery in EGFRmutant non-small cell lung cancer. Transl Lung Cancer Res 2019;8(Suppl 4):S391-S394. doi: 10.21037/tlcr.2019.09.09 